\title{
Opinion Expression Dynamics in Social Media Chat Groups: An Integrated Quasi-Experimental and Agent-Based Model Approach
}

\author{
Siyuan Ma $\mathbb{D}^{1}$ and Hongzhong Zhang $\mathbb{D}^{2}$ \\ ${ }^{1}$ Department of Communication, Michigan State University, East Lansing, MI, USA \\ ${ }^{2}$ School of Journalism \& Communication, Beijing Normal University, Beijing, China \\ Correspondence should be addressed to Hongzhong Zhang; zhanghz9@126.com
}

Received 25 July 2020; Revised 17 September 2020; Accepted 28 December 2020; Published 9 January 2021

Academic Editor: Hongshu Chen

Copyright (c) 2021 Siyuan Ma and Hongzhong Zhang. This is an open access article distributed under the Creative Commons Attribution License, which permits unrestricted use, distribution, and reproduction in any medium, provided the original work is properly cited.

\begin{abstract}
Social media chat groups, such as WeChat and WhatsApp groups, are widely applied in online communication. This research has conducted two studies to examine the individual level and collective level's opinion dynamics in those groups. The opinion dynamic is driven by two variables, people's perceived peer support and willingness of opinion expression. The perceived peer support influences the willingness of opinion expression, and the willingness influences the dynamics of real opinion-expression. First, the quasi-experimental study recruited twenty-five participants as the observation group and found that decreasing perceived peer support would significantly increase individuals' expression willingness to protect his/her opinion. To generalize the individual level findings to a collective level, the second study treated the social media chat groups as an undirected fullyconnected social network and simulated people's opinion expression dynamics with an agent-based model. The simulation indicated that (1) with the help of increased willingness of opinion expression, the minority opinion supporters as a collective did not fall silent but continue to express themselves and (2) increasing willingness of opinion expression would maintain the existence of minority opinion but could not help the minority reverse to the majority.
\end{abstract}

\section{Introduction}

Opinion discussions on social media have become increasingly diversified when accompanied by the progress of media technology, diversification of personal interests, and requirements of gratification [1]. This diversified feature boosts the formation of social media chat groups (short for social media groups), such as WhatsApp groups, WeChat groups, and Interest groups in online forums [2]. Each social media group has regular members and focuses on some specific topics [3]. By analyzing the number of active users monthly, social media groups have become increasingly popular and hold a broader influence on people's daily life [4]. Unlike open social media platforms that produce outbursts of information, social media groups provide an environment that allows a more in-depth discussion of opinions, which has a long-term impact on their users [5].
Therefore, the dynamic of opinion expression in social media groups is an essential topic and worth further study.

An ideal media environment should not hinder the expression of opinions, especially minority opinions. According to Habermas' public sphere theory [6], preserving space for the exchange of opinions helps maintain political legitimacy, social stability, and protect the welfare of citizens. Unfortunately, it is hard for the mass media to provide an ideal environment for opinions. For instance, opinions in mass media tended to grow more extreme $[7,8]$, and the expression of minority opinions tended to fall silent [9-11]. However, some recent studies proposed different findings that social media groups could provide a space for the expression of opinion, especially minority opinions (e.g., $[12,13])$. Thus, researchers design two studies with the following questions: 
(1) Quasi-experiment: is individuals' willingness of opinion expression protected in social media groups?

(2) Agent-based model simulation: how do individuals' willingness of opinion expression trigger the collective behavior, like the whole group's dynamics of opinion expression?

\section{Related Work}

2.1. Conflict Presumptions of Individual Opinion Expression Willingness: Spiral of Silence versus Corrective Action. Willingness of opinion expression refers to individuals' eagerness to share or post their opinions in public $[14,15]$. This term is widely used as a dependent variable in communication and political science studies. Previous studies have long been concerned that perceived peer support affects individuals' willingness of opinion expression. However, their primary two presumptions of that "effect" are in conflict with each other.

First, the spiral of silence theory suggests that the decrease of perceived peer support could lessen individuals' willingness of opinion expression, or even silence it because of people's fear of isolation $[9,16,17]$. The spiral of silence theory proposes that because individuals are afraid of being isolated, thus more perceived support will increase people's willingness of opinion expression, while less support will lessen the willingness $[18,19]$. This theory was proposed in the traditional mass media environment. Thus, the perceived support referred to support from mass media. Researchers later extended this support to peer relations and social media. Research by Oshagan [20] introduces the concept of the reference group, which is used as a synonym of peer, to describe family members, intimate partners, colleagues, and neighbors. Studies use this concept for reference and propose that reducing perceived peer support is even more effective than reducing mass media support when it tries to lessen individuals' willingness of opinion expression $[16,17,21]$.

On the contrary, the corrective action hypothesis proposes that controversial issues will inspire people to speak out their thoughts to change others' perceptions of public opinion [22]. When people perceive news or comments on social media going biased against them, they are concerned that the public may be swayed in that direction. Because of this fear, people are motivated to take action to correct public opinion by speaking out even when their opinion is in the minority [23]. Similar findings are found both in the news report and social media discussion situation; people who have received hostile news or received negative social media comments on their opinion would like to speak out to protect their opinion $[24,25]$.

Evidence suggests that whether audiences are motivated to keep silent or speak out depends on how they perceive others' opinions [26]. To deduct from the theory, when people feel the fear of isolation, the decrease of perceived peer support will lessen the willingness of opinion expression [27]. However, if people do not feel such fear, the perceived peer support will not diminish or even increase the willingness of opinion expression. Nowadays, people have more peers on social media (although they may never meet offline) comparing with the traditional mass media age; and they are more likely to find online peers who share the same opinions $[12,28,29]$. Because people are able to get psychological support conveniently from their online peers, they will not easily feel the fear of isolation [13]. Therefore, even though individuals perceive, they are the minority rather than the majority, their willingness of opinion expression may not decline but even increase. For instance, some studies found people who held the minority opinion did not have much change in their willingness of opinion expression while comparing with majority opinion $[15,30]$. Besides, some studies proposed that minority perception increased individuals' willingness of opinion expression [23-25, 29].

To sum up the findings above, there are conflicting conclusions between perceived peer support and willingness of opinion expression. Traditional mass media studies find that a decrease of support will lessen individuals' willingness of opinion expression $[9,16,17,20]$. However, recent social media studies have found that the perceived peer support will not decrease or even encourage this willingness (e.g., $[29,30]$. In the current study, social media groups created an unbiased fully-connected social network to help members get in touch with each other more conveniently, which suggests that people may not feel much fear of isolation. Therefore, this research proposes the first research question as follows:

RQ1. Will the decrease of perceived peer support enhance individuals' willingness of opinion expression in social media groups?

The current research has conducted a quasi-experiment (study 1) to answer this question. Details are presented later in this article.

Moreover, some other variables also influence the willingness of opinion expression. First, the spiral of silence theory [9] proposes hardcore to describe the people who are willing to express their opinions consistently regardless of social pressure. Studies have shown that if the hardcore is removed, minority opinion supporters' willingness to express will significantly decrease [31-33]. Furthermore, fear of authority, lack of self-efficiency, feeling the importance of the issue, accessibility of different opinions, and technical conditions of communication could all influence personal opinion expression $[13,33]$. In order to keep these variables consistent, the quasi-experiment applies repeated measurements on each individual.

\subsection{Simulating the Collective Opinion Expression Dynamics by} the Agent-Based Model. According to Sawyer's [34] mechanism of emergence, collective phenomena are collaboratively created by individuals yet may not reducible to individual action. Therefore, to generalize individual-level findings to the collective behavior of opinion expression in a social media chat group, we need an agent-based model (ABM) to simulate the dynamics. The ABM consists of agents, system space, and external environment. Agents have 
various attributes and are heterogeneous. ABM's rules act on the agents rather than the process of action [35, 36]. Each agent is autonomous and decides his/her behavior by learning memories, interacting with the neighbors, or the external environment with the rules of behavior [37]. To apply ABM in the current research, it will take into account people's initial opinion distribution in the social media group, the rules of opinion expression, and the evolution process. After setting up rules, new phenomena will emerge from ABM's simulation. Previous ABMs of opinion dynamics were originated from the Ising model. The two spin states of the Ising model corresponded to any relative set of concepts in social science, such as the concepts of right or wrong, and speaking out or silence. In principle, this model describes all multibody systems with two possible states [38]. Several representative evolutions of the Ising model are applied to the research of public opinion, such as the Voter model, Sznajd model, Majority-rule model, Krause-Hegselmann model, and Deffuant model $[39,40]$.

Although there were conflicting presumptions of the opinion expression willingness from individual-level studies, previous simulation studies mainly bought the idea of the spiral of silence, which proposed that less peer support would reduce the willingness and then also reduce or even silence the opinion expression. For instance, the study assumed people who received external opinion pressure larger than peer support would be less likely to speak out to protect their opinion. As time progressed, the minority opinion would fall into silence in any kind of social network, especially when it was the Barabasi-Albert scale-free network [41]. Social bot studies also proposed that the spiral of silence theory would lead agents to consensus opinion in a social network, with some agents being silenced. However, applying a small number of bots would easily shape the norms adopted by social media users and tip over the opinion climate [42].

The current research has found that those proposed models can be improved from two perspectives. First, we should analyze changes in opinion expression instead of changes of opinions. Some studies did not pay much attention to the difference between opinion expression and the opinion itself. From their assumption, every change of an agent would be assumed as the change of opinion itself, which referred an individual would change his or her opinion after receiving some influence from an opposite position (e.g., [35, 42, 43]). However, this assumption is not entirely consistent with the evidence. According to the spiral of silence theory and echo-chamber effect, the change of opinion does not happen in such a straightforward way. On the contrary, the opinion expression is relatively easier to change $[44,45]$. Besides, a silenced position does not necessarily mean the person has changed his/her idea. Secondly, we should use behavioral data to support the design of ABM, while previous studies' simulation did not have much support from real behavioral data. For instance, Sohn and Geidner [46] did distinguish the opinion expression and the opinion itself, interpreting the opinion dynamics more accurately. However, lacking real data intended that their model needed more parameters to adjust and thus reduced the validity [47]. Besides, previous models did not pay much attention to the possibility that the spiral of silence may not exist in the social media environment. Thus, behavioral data can help revise the rules of $A B M$.

In order to improve the two perspectives of model simulation mentioned in the previous paragraph, this study has planned to develop an agent-based model in study 2 that is consistent with quasi-experimental findings. Thus, the current research proposes as follows:

RQ2. How do individuals' change of opinion expression willingness influence the whole group's dynamics of real opinion-expression?

In order to clarify the relationship between study 1 and study 2, we provide a workflow. See Figure 1 for more details.

\section{Study 1: Quasi-Experiment in Social Media Group}

3.1. Methods. This study has designed a three-condition quasi-experiment to explore the relationship between perceived peer support and willingness of opinion expression from an individual level.

3.1.1. Participants. The quasi-experiment recruited $25 \mathrm{stu}-$ dents from a North China university to form the observation group. Those 25 participants all had the same opinions towards three similar questions. The quasi-experiment consisted of three conditions that the observation group had (1) more members, (2) evenly-matched members, or (3) fewer members when compared with the opposite opinion group. When the observation group members were more than the opposite opinion group, we named the observation group as a majority opinion condition; when the members were balanced, we named it as a balanced opinion condition; when the members were fewer than the opposite opinion group, we named it as a minority opinion condition. For each condition, we asked those people to discuss one of the three questions. The opposite opinion group contained different numbers of people in different conditions, and the current study recruited volunteers to form the opposite opinion group. Besides, those volunteering opposite group members were not the object of analysis.

3.1.2. Stimuli. The stimuli in this quasi-experiment were the proportion of the observation group in the total participants. When the proportion was high (which referred to majority opinion condition), we observed high perceived peer support; when the proportion was balanced (balanced opinion condition), we observed medium perceived peer support; when the proportion was low (minority opinion condition), we observed low perceived peer support. The manipulation check results are shown in the result part of study 1 .

The quasi-experimental conditions and participants' arrangements are shown in Table 1. 


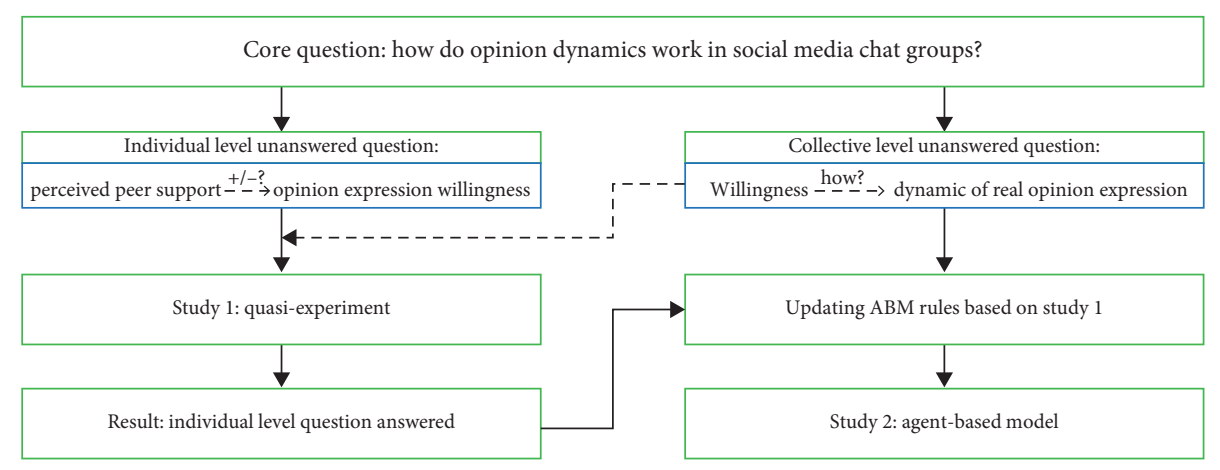

Figure 1: The workflow between study 1 and study 2 .

TABLE 1: Quasi-experimental participants arrangement.

\begin{tabular}{lccc}
\hline Condition & $\begin{array}{c}\text { Observation group } \\
\text { members }\end{array}$ & $\begin{array}{c}\text { Opposite opinion } \\
\text { members }\end{array}$ & $\begin{array}{c}\text { Expected level of perceived peer } \\
\text { support }\end{array}$ \\
\hline $\begin{array}{l}\text { Majority opinion (more observation members) } \\
\text { Balanced opinion (evenly-matched observation }\end{array}$ & $64 \%(N=25)$ & $36 \%(N=14)$ & High \\
members) & $53 \%(N=25)$ & $47 \%(N=22)$ & Medium \\
Minority opinion (fewer observation members) & $39 \%(N=25)$ & $61 \%(N=40)$ & Low \\
\hline
\end{tabular}

Note. The majority opinion situation refers that observation group members are more than the opposite opinion group; the balanced opinion situation refers that observation group members are evenly-matched with the opposite opinion group; the minority opinion situation refers that observation group members are fewer than the opposite opinion group.

3.1.3. Procedure. First, the researchers designed a survey with 15 questions, and each of them had two opposite opinions. All the questions were about the campus life in the college to make sure the participants had enough experience and background knowledge to discuss them. A total of 127 students from a North China University participated in the survey.

Second, 25 students who had the same opinion on three of those questions were recruited to form the observation group. The researcher announced that all participants who showed up in the experiment would be awarded the same research credits in the course, the credits were only related to whether they showed up in the experiment or not, not related to how active they behaved. Thus, even though the researcher could know how many comments each person had made in the discussion, the participants would not feel too much pressure of expressing themselves. Not being active does not impair their profits in this course.

Third, the researchers recruited volunteers who participated in the survey. They were assigned opinions that were opposed to the observation group. The researchers also provided credible materials so that the opposite opinion group members had enough knowledge to discuss with the observation group.

Fourth, the quasi-experiment manipulated the level of perceived peer support by changing the different conditions mentioned as follows: majority opinion, balanced opinion, and minority opinion condition. In each condition, we organized a group discussion which lasted approximately 30 minutes. Discussion topics were the three questions mentioned in the second step.

Finally, researchers controlled experimental conditions. In order to simulate the anonymous social media circumstance, each participant had a pseudoname. The pseudoname changed after each discussion. In addition, offline communication was not allowed. Therefore, an individual of the observation group only knew that some people supported his or her opinion, but could not match the people in the social media group to the offline. This design also helped to reduce the pressure of "you must speak out" from acquaintance friends.

Because of participants' personal reasons, three observation group members did not participate in the first condition, six members missed the second condition, and five members missed the third condition. The current study used an average interpolation method to handle the missing values.

3.1.4. Measurement. Perceived peer support and willingness of opinion expression all need operational definitions. In this quasi-experimental design, the two concepts are measured as follows:

(1) Perceived Peer Support. In a social media group, an individual's perceived peer support includes all comments from the same-opinion people. In traditional mass media, perceived peer support was hard to quantify. Researchers relied on self-reporting, asking how much the participant thought that other commenters could support his or her opinion with a Likert scale [14]. In this quasi-experiment, participants are not able to report their perceptions while participating in the discussion. However, all comments in the social media group are recorded so that we can use the real "received peer support" to represent the perceived support. Some evidence in 
previous studies supported this operationalization. For instance, youngsters who got less support from their partners would report less perceived peer support and exhibited more psychosocial adjustment problems [48]; and another psychological study about new mothers indicated that receiving more peer support would increase the perceptions of being trusted, accepted in the relationship [49]. Those studies support that received peer support can be a good predictor of perceived support. Therefore, perceived peer support is measured as a ratio as follows:

$$
\text { perceived peer support }=\frac{\text { the number of comments that an individual received from same opinion people }}{\text { the number of all comments }} .
$$

Note: "the number of all comments" should exclude comments made by this individual him/herself.

(2) Willingness of Opinion Expression. Similar to the perceived peer support, all comments in the group discussion are recorded. Therefore, the willingness of opinion expression is measured as the number of comments that an individual has posted during the discussion.

The current study created a workflow of doing study 1 . See Figure 2 for more details.

3.2. Results. The current study used a quasi-experiment to measure two variables and their relationships: perceived peer support and willingness of opinion expression. The research used individual-level repeated measurement.

3.2.1. Manipulation Check. The current study applied repeated measurement $F$-test to examine whether changing the situation of the majority opinion to a balanced opinion and a minority opinion would manipulate the perceived peer support or not.

The result in Table 2 depicted that all different types of $F$ test concluded that the variation of the majority opinion, balanced opinion, and minority opinion changed perceived peer support significantly.

The current study made pairwise comparisons to examine whether the direction of manipulation was correct or not. During the comparisons, 1 represented the majority opinion condition; 2 represented the balanced opinion condition; 3 represented the minority opinion condition.

This pairwise comparison in Table 3 concluded that, during the process from the majority condition to a balanced condition, the perceived peer support decreased nonsignificantly; during the process of the balanced to minority condition and from majority to minority, the perceived peer support decreased significantly. In general, it meant the perceived peer support was successfully decreased by changing an opinion group from the majority condition to a balanced condition and then to a minority condition. Therefore, the manipulation was acceptable.

3.2.2. Correlation Analysis. Repeated measurement correlation analysis (Rmcorr) is a method specifically designed to analyze the correlation between two variables under the repeated measurement condition [50]. This method is designed and efficiently reported within-group variance [50]. Because the current study measured each individual three times, the Rmcorr would be an appropriate tool to analyze the data. A Rmcorr $R$-package was prepared to measure the correlations between perceived peer support and willingness of opinion expression.

The results in Table 4 showed that when the observation group increased from the minority situation to a balanced situation, then to a majority situation, the correlation between perceived support and willingness was negative and significant $(r=-0.367, p=0.008)$. According to the $r$ and $p$ value, statistics indicated that the decrease of perceived peer support would significantly increase the willingness of opinion expression.

Moreover, the current study examined the relationship in two more concrete scenarios as follows: (1) when the balanced opinion situation increased to the majority opinion situation; (2) when the minority opinion situation increased to the balanced opinion situation.

Results in Table 5 showed that when the observation group increased from the balanced situation to a majority situation, the correlation between perceived support and willingness was negative and nonsignificant $(r=-0.200$, $p=0.327$ ). When the observation group increased from the minority situation to a balanced situation, the correlation between perceived support and willingness was negative and significant $(r=-0.604, p=0.001)$. According to the $r$ and $p$ value, statistics indicated that people in a minority opinion situation was more sensitive to the willingness of opinion expression rather than people in a majority opinion situation.

Therefore, the quasi-experiment concluded as (1) the decrease of perceived peer support would increase the willingness of opinion expression in a social media group and (2) members in a minority opinion were more sensitive to the change of peer support. When perceived less peer support, they were more willing to speak out rather than members in a majority opinion, or vice versa.

With the significant negative influence of perceived peer support towards the willingness of opinion expression, the research moved to a further step. Would individuals' change of opinion expression willingness influence a whole group's opinion expression dynamics? An agent-based model was designed to answer the question in the next study of the current research. 


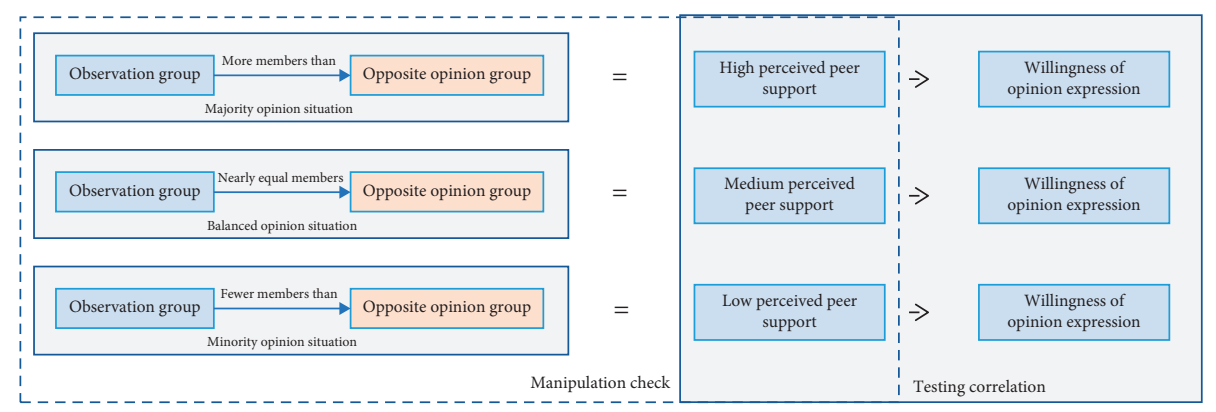

FIGURE 2: The workflow of study 1.

TABLE 2: The significant test of manipulating the level of perceived peer support.

\begin{tabular}{|c|c|c|c|c|}
\hline Source & Measurement & Mean square & $F$ & Sig. \\
\hline \multirow{4}{*}{ Perceived peer support } & Spherical distribution hypothesis & 0.124 & 10.489 & 0.000 \\
\hline & Greenhouse-Geisser & 0.171 & 10.489 & 0.001 \\
\hline & Huynh-Feldt & 0.162 & 10.489 & 0.001 \\
\hline & Lower limit & 0.248 & 10.489 & 0.004 \\
\hline
\end{tabular}

Note. Spherical distribution hypothesis, Greenhouse-Geisser, Huynh-Feldt, and lower limit refer to different measurement methods.

TABle 3: Pairwise comparison of manipulating the level of perceived peer support.

\begin{tabular}{|c|c|c|c|c|}
\hline Measurement & Opinion proportion & Mean difference & S.E. & Sig. b \\
\hline \multirow{3}{*}{ Perceived peer support } & $1 \longrightarrow 2$ & -0.055 & 0.042 & 0.621 \\
\hline & $1 \longrightarrow 3$ & $-0.152^{* * *}$ & 0.031 & 0.000 \\
\hline & $2 \longrightarrow 3$ & $-0.097^{* *}$ & 0.025 & 0.003 \\
\hline
\end{tabular}

Note. The significance level of the mean difference is 0.05 ; the adjusted multiple comparison method is Bonferroni ${ }^{* *} p<0.01 ;{ }^{* * *} p<0.001$.

\section{Study 2: Opinion Expression Dynamics with Agent-Based Model Simulation}

4.1. Modeling Opinion Expression Dynamics of a Social Media Group. This study has designed an ABM to simulate the opinion expression process and observe the collective level's emergent phenomena of two conflict opinion clusters' gambling. Specific operations and conclusions are detailed in the following sections.

4.1.1. Basic Rules of the ABM. Combined quasi-experiment conclusions with previous theories (e.g., corrective action) which mentioned opinion expression, and the study has summarized three basic rules to design the ABM.

(1) Once the opinions are determined, it is difficult to reverse them. The only factor that can change is the willingness of opinion expression, not the opinion itself.

(2) When people perceived their peer support is decreasing, they are more willing to speak out. The perceived peer support of one's opinion is measured
TABLE 4: The repeated measurement correlation between perceived peer support and willingness of opinion expression.

\begin{tabular}{lc}
\hline Item & Result \\
\hline$r$ (correlation) & $-0.367^{* * *}$ \\
degrees of freedom & 49 \\
$p$ value & 0.008 \\
${ }^{* * *} p<0.001$. &
\end{tabular}

TABLE 5: The repeated measurement correlation under balanced $\longrightarrow$ majority condition and minority $\longrightarrow$ balanced condition.

\begin{tabular}{lcc}
\hline & Balanced $\longrightarrow$ majority & Minority $\longrightarrow$ balanced \\
\hline Item & Result & Result \\
$r$ (correlation) & -0.200 & $-0.604^{* * *}$ \\
degrees of & 24 & 24 \\
freedom & 0.327 & 0.001 \\
$p$ value &
\end{tabular}

${ }^{* * * *} p<0.001$.

by the same ratio in the quasi-experiment that equals to

$$
P(\text { support })=\frac{\text { the number of comments that an individual received from the same opinion cluster }}{\text { the number of all comments }} .
$$


(3) Minority opinion cluster members are more sensitive to the change of perceived peer support while comparing with the majority.

4.1.2. Agents and Initial Assigned Parameters in the ABM. The study defined that all the agents were contained in the same chat group. Each agent represented a person in the group, and those agents could freely communicate with each other. In other words, this social media chat group was defined as an undirected and fully-connected social network. This network was separated into two opinion clusters, named $A$ and $B$. The two clusters had conflict opinions (assigned as opinion $=1$ or -1 ) towards each other. For each agent in one cluster, it also had an initial percentage of opinion expression willingness, which was randomly assigned by numbers ranging from $0 \%$ to $100 \%$ which is similar to Gaussian distribution.

$$
P \text { (willingness })=\text { random number }[0,1] \text {. }
$$

$0 \%$ represented that the agent did not have any willingness of opinion expression, while $100 \%$ represented that the agent must speak out when it was given a chance. The parameter cannot exceed 1 or below 0 at any time during the simulation. When it exceeds 1 or below 0 , it will be counted just as 1 or 0 . To be specific, the code set random number of $P$ (willingness) based on Gaussian distribution with $\mu=0.5$ and $\sigma=1$, if the random number of an agent exceeded 1 or below 0 , the code would randomly assign a new number to this agent, until it fell into the range of $[0,1]$. Thus, the final distribution of $P$ (willingness) is an approximately Gaussian distribution but with higher kurtosis. This is an acceptable assumption because social science discipline usually assumes human behavior concepts following Gaussian distribution. For instance, Chun and Lee's paper [14] used structural equation modeling (SEM) to analyze the relationship of perceived peer support and willingness to speak out, while the basic presumption of SEM is the two variables follow Gaussian distribution.

To sum up, the social media group was defined as a fully connected network and had two competing opinion clusters $A$ and $B$. For each agent in any cluster, it was assigned as an opinion and a percentage of opinion expression willingness. The assigned opinion could not change in the ABM, but the percentage of willingness could change over time.

4.1.3. Round of Speaking. The discussion in a real social media group could be conducted alternately by two conflict opinion clusters and formed a flowing stream of statements. In the $A B M$, the current study simulated the dynamics by defining round of speaking. The whole discussion process was segmented by several rounds of speaking. In each round, we randomly picked up several agents and asked if they chose to speak. The percentage of opinion expression willingness (which was defined in 4.1.2) was used here to determine whether the agent chose to speak or not. After one round of speaking, we moved to the next round.

4.1.4. Modeling Perceived Peer Support. The current study explicitly defined two types of perceived peer support. The first one was $p$ (initial support), which represented the initial perceived peer support. The $p$ (initial support) was operationalized as

$$
P(\text { initial support })=\frac{\text { the number of statements in the first } 15 \text { rounds from one opinion cluster (e.g., opinion } A \text { ) }}{\text { the number of all statements in the first } 15 \text { rounds }} \text {. }
$$

The second one was $p$ (later support), which represented the perceived peer support in the later one round of speaking. The $p$ (later support) was operationalized as

$$
P(\text { later support })=\frac{\text { the number of statements in one round from one opinion cluster (e.g., opinion } A)}{\text { the number of all statements in one round }} .
$$

When the agents in one opinion cluster found their $P$ (later support) was less than $P$ (initial support), that meant they perceived less peer support or vice versa.

\subsubsection{Modeling Willingness of Opinion Expression.} According to the basic rules, when agents perceive less peer support (than before), they will increase the willingness of opinion expression. On the contrary, when they perceive more peer support, they will decrease the willingness of opinion expression. Besides, minority opinion agents change their willingness broader than majority opinion agents. The current study defined the changing percentage of willingness as $p \%$ for minority agents, and the changing percentage of willingness as $q \%$ for majority agents. $p \%$ was larger than $q \%$ if there were one majority and one minority opinion clusters. On a special occasion, $p \%$ was equaled to $q \%$ if the two opinion clusters had the same number of agents.

4.1.6. The Procedure of ABM Simulation. Using the basic rules and parameters, let the agents work as follows: 
Step 1. Assumed that there was a social media group of 100 agents, some belong to opinion cluster $A$ and some belong to $B$.

Step 2. Randomly assigned initial $P$ (willingness) to each agent with a similar Gaussian distribution. For instance, suppose $A$ was the majority opinion and $B$ was the minority opinion, $A_{i}(1,30 \%)$ meant an agent in cluster $A$ was assigned to opinion $=1$ and $P$ (willingness $)=30 \%$ possibility to speak out when given a chance. $B_{j}(-1,50 \%)$ meant an agent in cluster $B$ was assigned to an opposite opinion $=-1$ and $P$ (willingness $)=50 \%$ possibility to speak out when given a chance.

Step 3. Set 100 rounds of speaking, and in each round, we randomly selected $N=10$ agents. Whether the ten agents chose to speak or not were depended on their percentage of opinion expression willingness. For instance, if $A_{i}(1,30 \%)$ was one of the 10 agents selected in one round, it would have a $30 \%$ possibility to speak out in that round. Here, the 100 rounds of speaking are used to simulate a real discussion time of a topic. Even though it is possible that, after several thousands of discussion rounds, later the majority and minority situations are switched, it is less possible to have such a long-lasting discussion in the real world.

Step 4. The simulation ran 15 rounds first, then calculated $p$ (initial support) of opinion $A$ and opinion $B$.

Step 5. Starting from round 16, for each round, if one opinion cluster's perceived peer support changed comparing with the total first 15 rounds, we had four situations:

$A$. The opinion cluster was the minority and the peer support decreased. Those agents would increase their opinion expression willingness by $p \%$. For instance, if cluster $B$ found

$$
\begin{gathered}
P \text { (later support })<P(\text { initial support }), \\
\text { then } B_{j}(t+1)=\left(-1,50^{*}(1+p) \%\right) .
\end{gathered}
$$

$B$. The opinion cluster was the majority and the peer support decreased. Those agents would increase their opinion expression willingness by $q \%$. For instance, if cluster $A$ found

$$
\begin{gathered}
P \text { (later support })<P(\text { initial support }), \\
\text { then } A_{i}(t+1)=\left(1,30^{*}(1+q) \%\right) .
\end{gathered}
$$

C. The opinion cluster was the minority and the peer support increased. Those agents would decrease their opinion expression willingness by $p \%$. For instance, if cluster $B$ found

$$
\begin{gathered}
P(\text { later support })>P(\text { initial support }), \\
\text { then } B_{j}(t+1)=\left(-1,50^{*}(1-p) \%\right) .
\end{gathered}
$$

$D$. The opinion cluster was the majority and the peer support increased. Those agents would decrease their opinion expression willingness by $q \%$. For instance, if cluster $A$ found

$$
\begin{gathered}
P(\text { later support })>P(\text { initial support }), \\
\text { then } A_{i}(t+1)=\left(1,30^{*}(1-q) \%\right) .
\end{gathered}
$$

Once determined an opinion, people in the group would not change their views. The most extreme possibility was the opinion remained unchanged, but the percentage of opinion expression willingness was $0 \%$ or $100 \%$.

This study used a $\mathrm{C}++$ program to run the ABM.

4.2. Results. Three key parameters were needed to adjust in the $\mathrm{ABM}$ as follows: the percentage of opinion expression willingness $p \%$ and $q \%$, and the number of agents in two opinion clusters: $a$ and $100-a$. This simulation set several levels of each parameter: When $a=10,20,30$, and $40,(p \%, q$ $\%)=(5 \%, 2.5 \%),(10 \%, 5 \%),(15 \%, 7.5 \%)$, and $(20 \%, 10 \%)$. When $a=50,(p \%, q \%)=(5 \%, 5 \%),(10 \%, 10 \%),(15 \%, 15 \%)$, and $(20 \%, 20 \%)$. Therefore, this simulation had $5 * 4=20$ in different situations. The ABM in each situation ran 100 times and took the average to construct the curve of two clusters' opinion expression.

\subsubsection{Model Analysis When Two Opinions Were Balanced.} To simulate the situation that two opinions were balanced, this research assigned two opposite opinions, $A$ and $B$, and each of them had 50 agents. Their initial willingness of opinion expression was a randomly assigned normal distribution before the simulation. Following the rules mentioned above, the ABM drew the opinion expression percentage of opinion A. The opinion expression percentage was operationalized as

$$
P(\text { expression })=\frac{\text { the number of statements in one round from one opinion cluster }(\text { e.g., opinion } A)}{\text { the number of all statements in one round }} .
$$

Note that the opinion expression percentage is counted by statements made by agents, which is different from the willingness of opinion expression in people's minds. If at a specific time point, the opinion expression percentage of cluster $A$ equaled to $50 \%$, that meant opinion cluster $A$ agents made $50 \%$ of the statements in the discussion.

From Figure 3, the current study concluded that the two equally balanced opinions antagonized one another and 

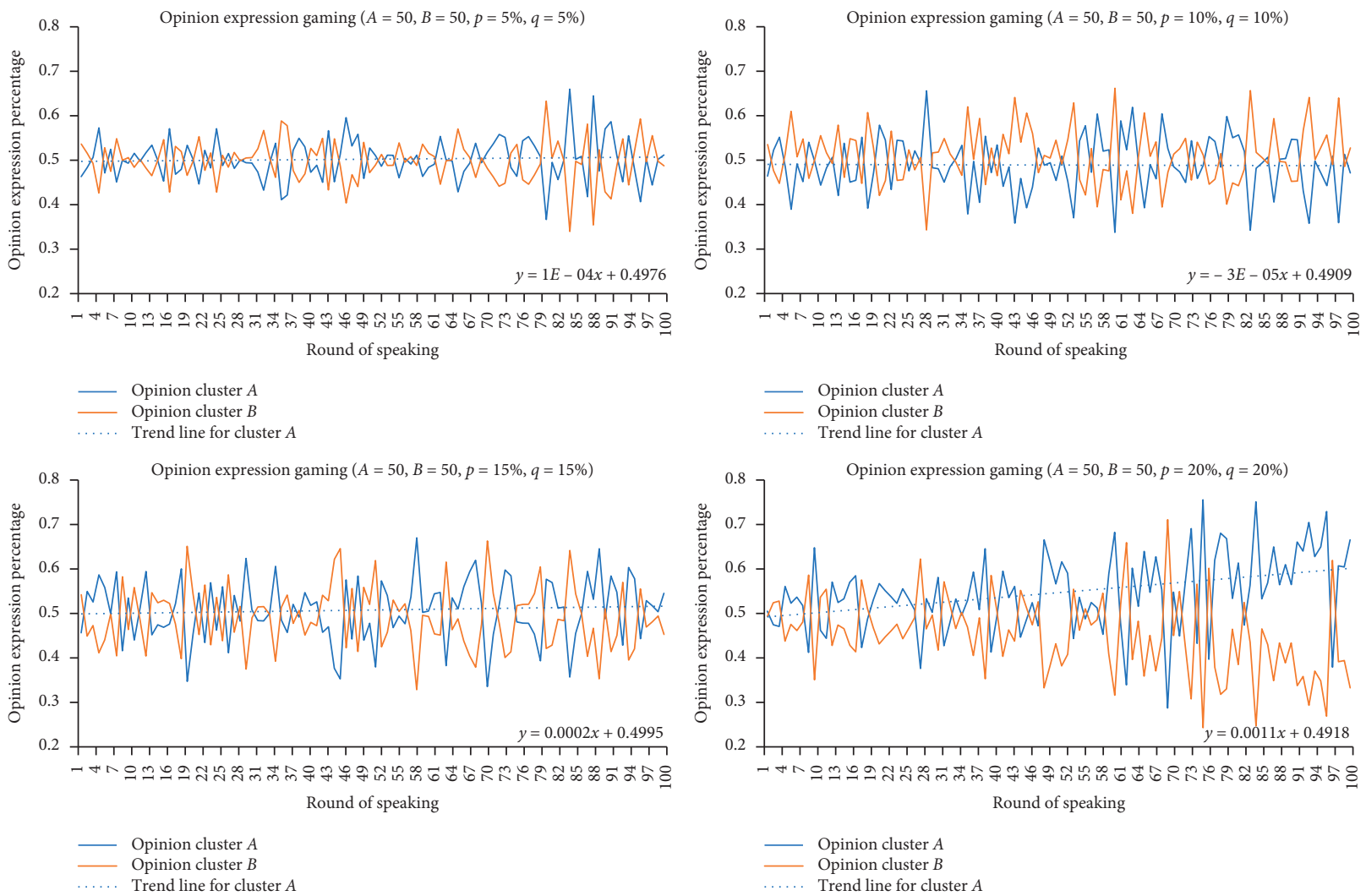

Figure 3: Balanced opinion situation $(A=50$ and $B=50)$. Note. Opinion expression percentage, with $A$ (minority cluster) $=$ blue thick line and $B$ (majority cluster) $=$ red thin line. Round of speaking represents time because each discussion allows 100 rounds of speaking. All the results were shown in the average score of 100 times' simulation.

were always fluctuating up and down around $50 \%$. Different values of the parameter $p \%(5,10$, and 15$)$ shared similar trends. However, when the parameter $p \%$ gets larger to $20 \%$, the opinion expression percentage of opinion A could fluctuate to $60 \%$ at the end of 100 rounds of speaking, which is more unstable than small $p \%$ values.

\subsubsection{Model Analysis When the Social Media Group Was} Divided into Two Opinions: One Majority and One Minority. To simulate the situation that two opinions had one minority and another majority, this research assigned two opposite opinions, $A$ and $B$, with four situations: $A=10, B=90$; $A=20, B=80 ; A=30, B=70 ; A=40, B=60$. Each agent's initial willingness of opinion expression was a proportion that was randomly assigned with normal distribution before the simulation. Following the rules mentioned above, the simulation ran 100 times in each situation and then drew the opinion expression percentage on the minority/majority opinion discussion in Figure 4.

In Figure 4, the blue thick line referred to the minority opinion; the red thin line referred to the majority opinion. From the figure, the current study indicated that the two opinions antagonized one another, and the minority opinion spoke out more than expected. For instance, the minority opinion members constituted $10 \%$ of the population, while constituted $15-25 \%$ in opinion expression at the end of 100 rounds of speaking. Different values of parameter $p \%$ shared the same trends. Besides, the increasing range of minority opinion was enhanced by parameter $p \%$.

However, with the increasing members of the minority opinion, the opinion expression percentage became closer to the percentage of the population. For instance, in Figure 5, when the minority population increased from 10 to 40 , the expression percentage did not increase continuously above $40 \%$ but fluctuated around $40 \%$ instead. Different values of parameter $p \%$ shared the same trends.

Figures 6 and 7 show the simulation results when $A=20$ and 30. Their opinion dynamics fluctuated less than the position in Figure 4 but more than the position in Figure 5 .

To sum up, according to the rules of $\mathrm{ABM}$, the minority opinion's expression percentage behaved better than the percentage of the total population when the opinion had very few supporters. For instance, when $A=10$ and $B=90$, the opinion expression percentage could be around $15 \%-$ $25 \%$ for cluster $A$, but only $75 \%-85 \%$ for cluster $B$. The larger the number of parameters $p$ and $q$, the more unstable the trend would be. However, we could not find enough support that the minority opinion could be reversed to be the majority opinion. As the number of minority opinion members increased, the superiority of opinion expression gradually declined. 

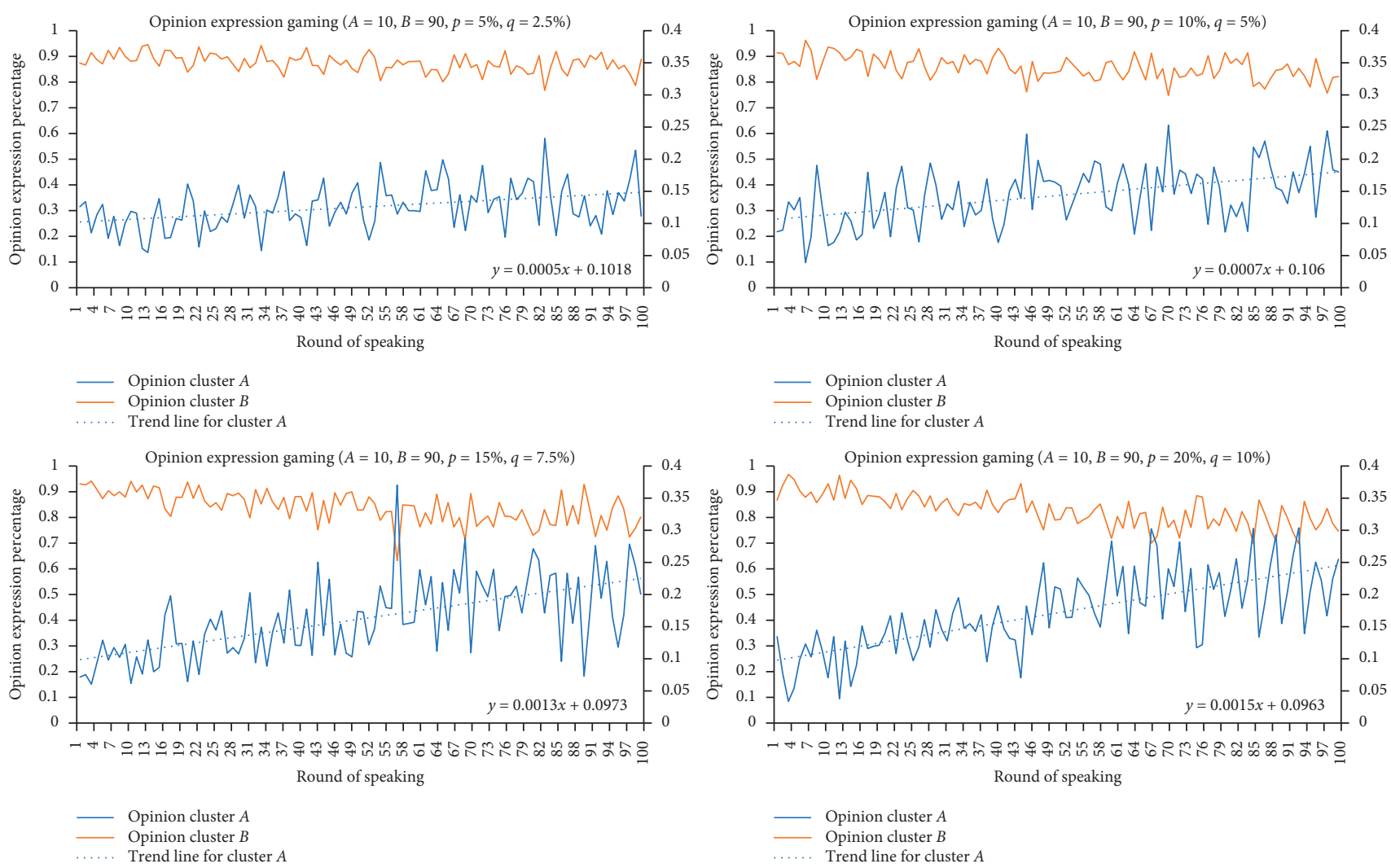

Figure 4: Minority/majority opinion expression. (For example, $A=10, B=90$ ). Note. Opinion expression percentage, with $A$ (minority cluster $)=$ blue thick line and $B$ (majority cluster) $=$ red thin line. Round of speaking represents time because each discussion allows 100 rounds of speaking. All the results were shown in the average score of 100 times' simulation.
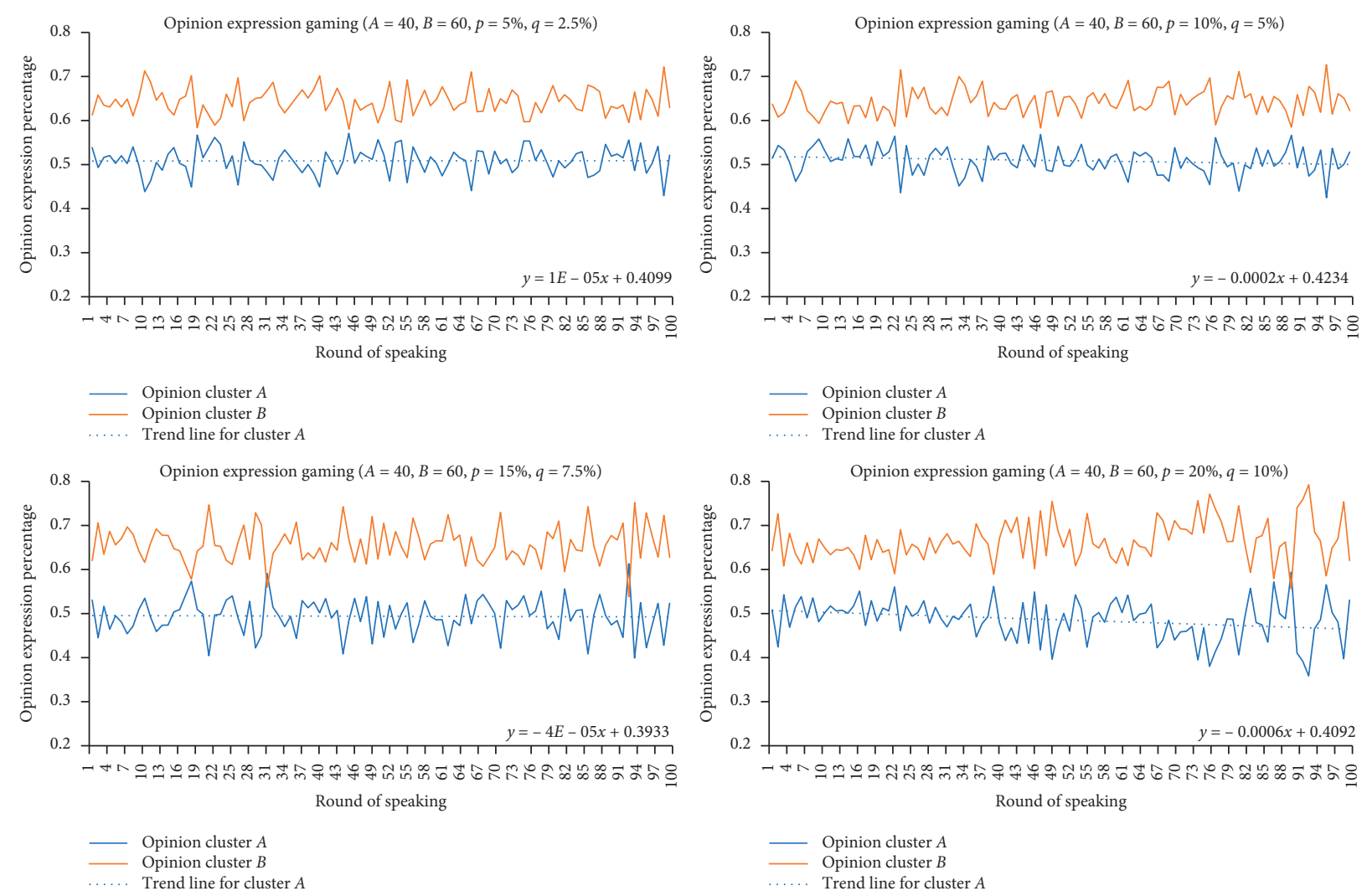

FIgURe 5: Minority/majority opinion expression. (For example, $A=40, B=60$ ). Note. Opinion expression percentage, with $A$ (minority cluster) = blue thick line and $B$ (majority cluster) $=$ red thin line. Round of speaking represents time because each discussion allows 100 rounds of speaking. All the results were shown in the average score of 100 times' simulation. 

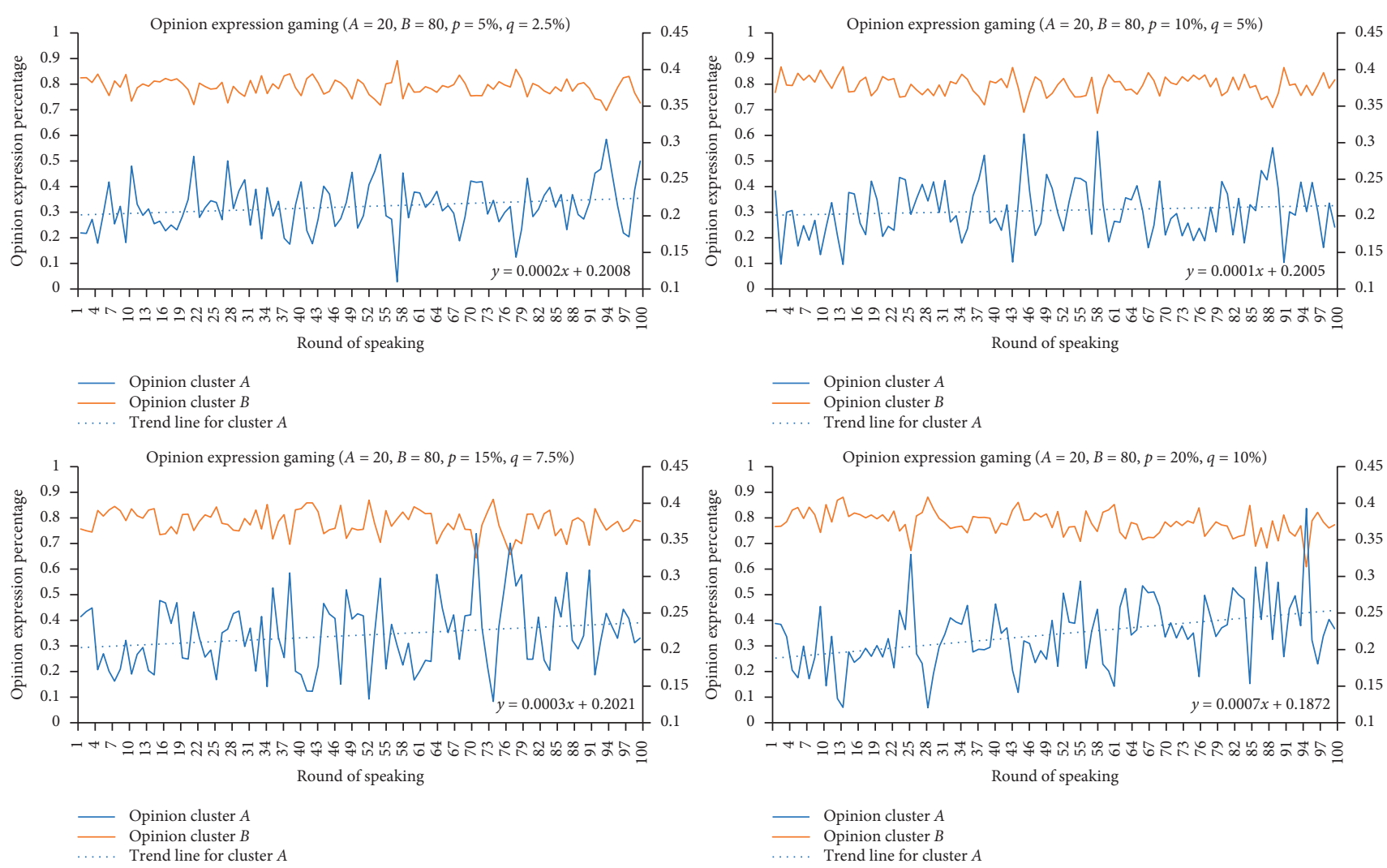

Figure 6: Minority/majority opinion expression. (For example, $A=20, B=80$ ). Note. Opinion expression percentage, with $A$ (minority cluster) = blue thick line and $B$ (majority cluster) = red thin line. Round of speaking represents time because each discussion allows 100 rounds of speaking. All the results were shown in the average score of 100 times' simulation.
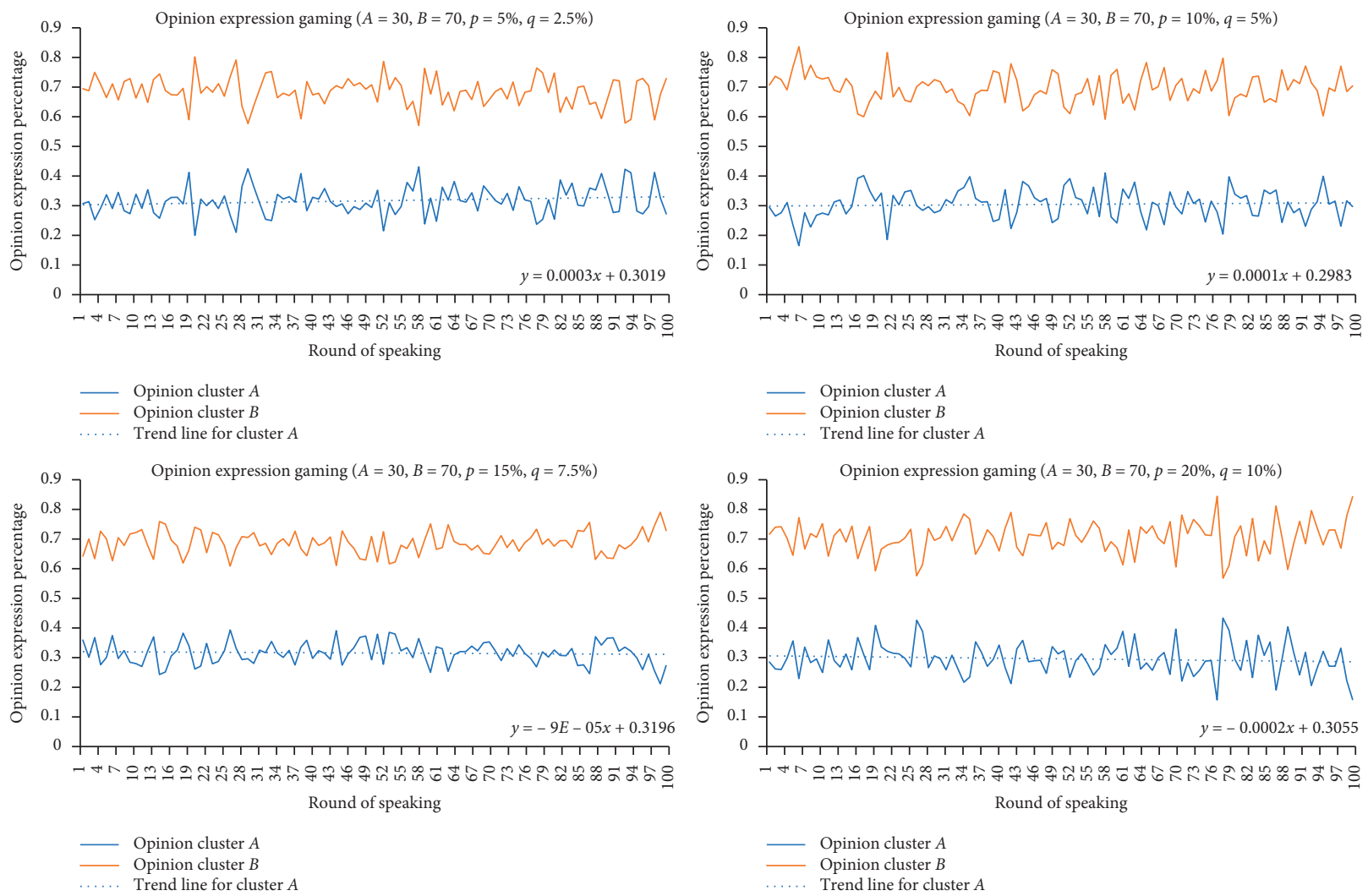

Figure 7: Minority/majority opinion expression. (For example, $A=30, B=70$ ). Note. Opinion expression percentage, with $A$ (minority cluster) = blue thick line and $B$ (majority cluster) = red thin line. Round of speaking represents time because each discussion allows 100 rounds of speaking. All the results were shown in the average score of 100 times' simulation. 


\section{Conclusion and Discussion}

The current research concluded that the minority opinion members were more sensitive to the change of peer support than the majority opinion members. Notably, an individual's willingness of opinion expression could be encouraged while the perceived peer support decreased, which was significantly different from the traditional spiral of silence studies as well as the simulation studies. One potential explanation of this finding is, the fear of isolation is the premise of the spiral of silence [27], while people tend to find peers in social media groups, so they may not feel enough isolated even if they receive less support. Besides, the studies of corrective action and hostile media have proposed that when people feel their opinions are unfairly suppressed, they will feel more indignation and then protecting their opinions rather than perceiving fear $[51,52]$. Thus, less support in social media groups can encourage the willingness of opinion expression.

New phenomena emerged during the simulation of opinion expression dynamics in the social media group. The simulation model based on the quasi-experimental results showed that the increase of the willingness of opinion expression on an individual level could encourage the real opinion expression and prevent the whole cluster of minority opinion from falling into silence. That means it is quite difficult to achieve complete suppression of one opinion in social media groups. However, findings also indicate that enhancing willingness of opinion expression is not a panacea for the minority opinion members to demand greater influence. Because results showed that the minority's opinion expression percentage could only behave relatively better than the minority opinion supporters' population percentage, then floated up and down. Reaching the final one-side win or reversing the minority opinion to the majority may both need time and luck, which is hard to achieve in real life.

The current research findings have practical implications. First, people should be vigilant if some specific interest groups intend to manipulate their willingness of opinion expression. Because it can help seek greater influence not matching their capacity and then mislead the public opinion to be more beneficial to themselves. Second, those who support a minority opinion should have the confidence to survive in social media groups. Third, it is probably not necessary for majority opinion members to pursue a complete suppression of opinions; on the contrary, it is necessary to acknowledge that most changes of pluralistic public opinions are ordinary and respectful. Finally, social media chat groups are widely existed nowadays but are different from those open social media platforms. Therefore, more researches are encouraged to study further about the cause, effect, and mechanism of opinion dynamics in social media groups.

Here are some limitations of the current research. First, in order to maintain a good external validity of the results, the quasi-experiment tries to simulate a real environment of a social media chat group and uses behavioral data to operationalize psychological concepts. However, it does not test the difference between intention and behavior, such as perceived peer support and received peer support, willingness to speak out, and the actual speak out. We would suggest future studies to come up with a better design to measure the intensions. Second, the quasi-experiment needs more participants in the observation group; and the percentage changes of the observational group between the majority, balance, and minority situations need to be controlled more strictly. Third, we do not have enough empirical data to support that people's willingness of opinion expression is normally distributed, but the researchers assume that, it is in the agent-based model. Although it is a relatively common assumption that human behavior is following Gaussian distribution, it could have possibilities and may cause bias in the simulation. Therefore, researchers plan to do further studies focusing on exploring more theoretically-supported communication strategies to simulate the collective level phenomenon and design more rigorous experiments to test the individual level presumptions.

\section{Data Availability}

The quasi-experimental data used to support the findings of this study are available from the corresponding author upon request. The agent-based model codes are included within the supplementary information file.

\section{Conflicts of Interest}

The authors declare that they have no conflicts of interest.

\section{Supplementary Materials}

The supplementary file includes codes of conducting the agent-based model. (Supplementary Materials)

\section{References}

[1] M. Jing and C. Zang, "On the information flow mode, demassification communication and the influence of mass media over the social cohesion under the age of media convergence," Journalism \& Communication, vol. 5, pp. 3442, 2011.

[2] Y. Chen, "Comparison of communication mechanism between micro-blog and WeChat under different identity," Press Circles, vol. 3, pp. 54-57, 2015.

[3] G. M. Yu, "Some ideas about the future development of the media industry," News Front, vol. 1, 2014.

[4] K. N. Hampton, I. Shin, and W. Lu, "Social media and political discussion: when online presence silences offline conversation," Information, Communication \& Society, vol. 20, no. 7, pp. 1090-1107, 2017.

[5] Z. A. Zhang and K. R. Shu, "Research on the public opinion of WeChat: relationship network and ecological characteristics," Shanghai Journalism Review, vol. 20, no. 6, pp. 29-37, 2016.

[6] J. Habermas, The Structural Transformation of the Public Sphere, Xue Lin Publishing House, Shanghai, China, 1962.

[7] D. Goldie, M. Linick, H. Jabbar, and C. Lubienski, "Using bibliometric and social media analyses to explore the "echo chamber" hypothesis," Educational Policy, vol. 28, no. 2, pp. 281-305, 2014. 
[8] G. H. Guo, "On the "group polarization" tendency of the main body of network public opinion," Journal of Social Science of Hunan Normal University, vol. 6, pp. 110-113, 2004.

[9] E. Noelle-Neuman, The Spiral of Silence: Public Opinion-Our Social Skin, Peking University Press, Bejing, China, 1974.

[10] D. A. Scheufele and P. Moy, "Twenty-five years of the spiral of silence: a conceptual review and empirical outlook," International Journal of Public Opinion Research, vol. 12, pp. 3-28, 2000.

[11] L. Willnat, WP. Lee, and B. H. Detenber, "Individual-level predictors of public outspokenness: a test of the spiral of silence theory in Singapore," International Journal of Public Opinion Research, vol. 14, no. 4, pp. 391-412, 2002.

[12] M. J. Lee and J. W. Chun, "Reading others' comments and public opinion poll results on social media: social judgment and spiral of empowerment," Computers in Human Behavior, vol. 65, pp. 479-487, 2016.

[13] G. Neubaum and N. C. Krämer, "Opinion climates in social media: blending mass and interpersonal communication," Human Communication Research, vol. 43, no. 4, pp. 464-476, 2017.

[14] J. W. Chun and M. J. Lee, "When does individuals' willingness to speak out increase on social media? Perceived social support and perceived power/control," Computers in Human Behavior, vol. 74, pp. 120-129, 2017.

[15] T. Zerback and N. Fawzi, "Can online exemplars trigger a spiral of silence? Examining the effects of exemplar opinions on perceptions of public opinion and speaking out," New Media \& Society, vol. 19, no. 7, pp. 1034-1051, 2017.

[16] A. F. Hayes, "Exploring the forms of self-censorship: on the spiral of silence and the use of opinion expression avoidance strategies," Journal of Communication, vol. 57, no. 4, pp. 785-802, 2007.

[17] P. Moy, D. Domke, and K. Stamm, "The spiral of silence and public opinion on affirmative action," Journalism \& Mass Communication Quarterly, vol. 78, no. 1, pp. 7-25, 2001.

[18] E. Katz, "Publicity and pluralistic ignorance: notes on "the spiral of silence"," in Public Opinion and Social Change. For Elisabeth Noelle-Neumann, H. Baier, Kepplinger, and K. Reumann, Eds., pp. 28-38, Westdeutscher Verlag, Wiesbaden, Germany, 1981.

[19] E. Wartella, C. Whitney, Mass Communication Review Yearbook, Sage, Vol. 4, Beverley Hills, CA, USA, 1983.

[20] H. Oshagan, "Reference group influence on opinion expression," International Journal of Public Opinion Research, vol. 8, no. 4, pp. 335-354, 1996.

[21] C. J. Glynn, A. F. Hayes, and J. Shanahan, "Perceived support for one's opinions and willingness to speak out: a metaanalysis of survey studies on the "spiral of silence"," Public Opinion Quarterly, vol. 61, no. 3, pp. 452-463, 1997.

[22] W. P. Davison, "The third-person effect in communication," Public Opinion Quarterly, vol. 47, no. 1, pp. 1-15, 1983.

[23] H. Rojas, "“Corrective" actions in the public sphere: how perceptions of media and media effects shape political behaviors," International Journal of Public Opinion Research, vol. 22, no. 3, pp. 343-363, 2010.

[24] M. Duncan and D. Coppini, "Party v. The People: testing corrective action and supportive engagement in a partisan political context," Journal of Information Technology \& Politics, vol. 16, no. 3, pp. 265-289, 2019.

[25] M. Duncan, A. Pelled, D. Wise et al., "Staying silent and speaking out in online comment sections: the influence of spiral of silence and corrective action in reaction to news," Computers in Human Behavior, vol. 102, pp. 192-205, 2020.
[26] Y. Tsfati, N. J. Stroud, and A. Chotiner, "Exposure to ideological news and perceived opinion climate: testing the media effects component of spiral-of-silence in a fragmented media landscape," The International Journal of Press/Politics, vol. 19, no. 1, pp. 3-23, 2014.

[27] P. J. Shoemaker, M. Breen, and M. Stamper, "Fear of social isolation: testing an assumption from the spiral of silence," Irish Communication Review, vol. 8, pp. 65-78, 2000.

[28] N. Pang, S. S. Ho, A. M. R. Zhang, J. S. W. Ko, W. X. Low, and K. S. Y. Tan, "Can spiral of silence and civility predict click speech on Facebook?" Computers in Human Behavior, vol. 64, pp. 898-905, 2016.

[29] G. W. Yun, S.-Y. Park, and S. Lee, "Inside the spiral: hostile media, minority perception, and willingness to speak out on a weblog," Computers in Human Behavior, vol. 62, pp. 236-243, 2016.

[30] C. Wells, K. J. Cramer, M. W. Wagner et al., "When we stop talking politics: the maintenance and closing of conversation in contentious times," Journal of Communication, vol. 67, no. 1, pp. 131-157, 2017.

[31] C. J. Glynn and J. M. McLeod, "Implication of the spiral of silence theory for communication and public opinion research," in Political Communication Yearbook, D. R. Sanders, L. L. Kaid, and D. Nimmo, Eds., pp. 43-65, Southern Illinois University Press, Carbondale, IL, USA, 1984.

[32] D. G. Mcdonald, C. J. Glynn, S.-H. Kim, and R. E. Ostman, "The spiral of silence in the 1948 presidential election," Communication Research, vol. 28, no. 2, pp. 139-155, 2001.

[33] L. Willnat, "Mass media and political outspokenness in Hong Kong: linking the third-person effect and the spiral of silence," International Journal of Public Opinion Research, vol. 8, no. 2, pp. 187-212, 1996.

[34] R. K. Sawyer, “The mechanisms of emergence," Philosophy of the Social Sciences, vol. 34, no. 2, pp. 260-282, 2004.

[35] M. Pineda and G. M. Buendía, "Mass media and heterogeneous bounds of confidence in continuous opinion dynamics," Physica A: Statistical Mechanics and Its Applications, vol. 420, pp. 73-84, 2015.

[36] W. B. Wang, "The present situation and prospect of the research on evolutionary game theory," Statistics and Decision, vol. 25, no. 3, pp. 158-161, 2009.

[37] N. Jung, E. S. Cho, J. H. Choi, and J. W. Lee, "Agent-based models in social physics," Journal of the Korean Physical Society, vol. 72, pp. 1272-1280, 2018.

[38] Z.-D. Zhang, "Conjectures on the exact solution of threedimensional (3D) simple orthorhombic Ising lattices," Philosophical Magazine, vol. 87, no. 34, pp. 5309-5419, 2007.

[39] F. W. S. Lima, "Three-state majority-vote model on square lattice," Physica A: Statistical Mechanics and Its Applications, vol. 391, no. 4, pp. 1753-1758, 2012.

[40] Y. J. Liu, Q. Li, and W. Y. Niu, "A summary of the model of public opinion dynamics," Management Review, vol. 25, no. 1, pp. 167-176, 2013.

[41] Y. Wu, Y.-J. Du, X.-Y. Li, and X.-L. Chen, "Exploring the spiral of silence in adjustable social networks," International Journal of Modern Physics C, vol. 26, no. 11, Article ID 1550125, 2015.

[42] B. Ross, L. Pilz, B. Cabrera, F. Brachten, G. Neubaum, and S. Stieglitz, "Are social bots a real threat? An agent-based model of the spiral of silence to analyse the impact of manipulative actors in social networks," European Journal of Information Systems, vol. 28, no. 4, pp. 394-412, 2019.

[43] L. Deng, Y. Liu, and F. Xiong, "An opinion diffusion model with clustered early adopters," Physica A: Statistical 
Mechanics and Its Applications, vol. 392, no. 17, pp. 35463554, 2013.

[44] A. Boutyline and R. Willer, "The social structure of political echo chambers: variation in ideological homophily in online networks," Political Psychology, vol. 38, no. 3, pp. 551-569, 2017.

[45] D. Trilling, M. van Klingeren, and Y. Tsfati, "Selective exposure, political polarization, and possible mediators: evidence from The Netherlands," International Journal of Public Opinion Research, vol. 29, pp. edw003-213, 2017.

[46] D. Sohn and N. Geidner, "Collective dynamics of the spiral of silence: the role of ego-network size," International Journal of Public Opinion Research, vol. 28, no. 1, pp. 25-45, 2015.

[47] J. J. H. Zhu, Y. Huang, and X. Zhang, "Dialogue on computational communication research: origins, theories, methods, and research questions," Communication \& Society, vol. 44, pp. 1-24, 2018.

[48] P. L. East, L. E. Hess, and R. M. Lerner, "Peer social support and adjustment of early adolescent peer groups," The Journal of Early Adolescence, vol. 7, no. 2, pp. 153-163, 1987.

[49] C.-L. Dennis, "Postpartum depression peer support: maternal perceptions from a randomized controlled trial," International Journal of Nursing Studies, vol. 47, no. 5, pp. 560-568, 2010.

[50] J. Z. Bakdash and L. R. Marusich, "Repeated measures correlation," Frontiers in Psychology, vol. 8, p. 456, 2017.

[51] C. S. Carver, "Negative affects deriving from the behavioral approach system," Emotion, vol. 4, no. 1, pp. 3-22, 2004.

[52] H. Hwang, Z. Pan, and Y. Sun, "Influence of hostile media perception on willingness to engage in discursive activities: an examination of mediating role of media indignation," Media Psychology, vol. 11, no. 1, pp. 76-97, 2008. 